\title{
Computer system for reconstructing and analyzing random structural models of protein-protein interaction networks
}

\author{
N.L. Podkolodnyy ${ }^{1,2 *}$, D.A. Gavrilov ${ }^{3}$, O.A. Podkolodnaya ${ }^{1}$ \\ ${ }^{1}$ Institute of Cytology and Genetics SB RAS, Novosibirsk, Russia \\ ${ }^{2}$ Institute of Computational Mathematics and Mathematical Geophysics SB RAS, Novosibirsk, Russia \\ ${ }^{3}$ Novosibirsk State University, Novosibirsk, Russia \\ *e-mail:pnl@bionet.nsc.ru
}

Key words: protein interaction network analysis, random graph models, circadian rhythm

Motivation and Aim: Modern experimental technologies in molecular biology allow the reconstruction of various types of biological networks, including gene and metabolic networks, interaction networks, gene co-expression networks, a network of diseases, etc. In this regard, the development of methods and tools for constructing structural random models, preserving the structural and functional features of the biological network, is an urgent task of computer systems biology. The structural random models are needed to search for patterns of the structural and functional organization of biological networks, to study their influence on the function of biological networks, to test various statistical hypotheses on networks, to search for network biomarkers, and so on.

Methods and Algorithms: The Markov chain simulation method was used for generating connected random graphs that preserve the structural features of the biological network. The computational server asynchronously executes a client's (Cytoscape plugin's) request for reconstruction of structural random models of biological networks using the software libraries Random Network Generator [1] and new our method based on GTrie Scanner.

Results: We developed the computer system for reconstructing structural random models of biological networks preserving the following structural characteristics: the distribution of vertex degrees, the joint degree distribution, the average degree of neighboring vertices, the clustering coefficient, the clustering spectrum, the frequencies of different sizes structural motifs, etc. The Cytoscape plugin provides the loading the biological network for analyzing, making a request to the remote computational server for the reconstruction of various structural models according to their specification, visualize of reconstructed structural models and their comparative analysis in Cytoscape package [2]. The reconstruction of the structural models of protein-protein interactions (PPI) in mouse liver and their comparative analysis allowed us to identify the circadian dynamics of structural and functional regularities of PPI networks.

Conclusion: The system developed by us allows us to build random models of biological networks and to analyze them for searching the patterns of the structural and functional organization of biological networks, to test various statistical hypotheses on networks, to search for network biomarkers, and so on.

Acknowledgements: Supported by Presidium of SD RAS (No. 0324-2018-0021) and by RF Government (No. 0324-2018-0017).

\section{References}

1. Random Network Generator: https://github.com/polcolomer/RandNetGen

2. Shannon P. et al. (2003) Cytoscape: a software environment for integrated models of biomolecular interaction networks. In Genome research. 13(11):2498-2504. 